\title{
Electronic Medical Records: Provotype visualisation maximises clinical usability
}

\author{
David Pao \\ School of Design \\ Royal College of Art \\ London, SW7 2EU, UK \\ david.pao@network.rca.ac.uk
}

\author{
John Stevens \\ School of Design \\ Royal College of Art \\ London, SW7 2EU, UK \\ john.stevens@rca.ac.uk
}

Netta Weinstein

School of Psychology

Cardiff University

Cardiff, CF10 3AT, UK

weinsteinn@cardiff.ac.uk

\author{
Dan Lockton \\ School of Design \\ Carnegie Mellon University \\ Pittsburgh, PA 15213, USA \\ danlockton@cmu.edu
}

\begin{abstract}
The Electronic Medical Record (EMR) is the essential tool of the clinical consultation, effectively replacing the paper medical record. Since its gradual adoption in the early 2000 s there has been a failure to achieve even moderate levels of EMR usability in clinical settings, resulting in a negative impact on clinical care, time efficiency and patient safety. This research explores how deeper collaboration with clinical users through participatory design, drawing on the disciplines of visual design, user experience (UX) design and visual analytics, might offer a more effective approach to this important problem. The lead researcher for this project is both a practising doctor and design researcher. Usability of two commercial EMR interfaces in the field of sexual health is explored through a mixed method survey, with responses used to inform the design of an interface provotype. This in turn is evaluated through repeat survey and 'test-drive' talk-aloud workshops. Results from the survey on two commercial EMR interfaces $(n=49)$ revealed deep dissatisfaction particularly around issues of navigation, flow of consultation, frustration, safety, time-dependent and time-independent data, data complexity and data salience. Comparative provotype evaluation $(n=63)$ showed that clinically-relevant visualisation offers marked gains in clinical usability and performance. This research argues for a re-imagining of the way we look at medical data during the clinical consultation so that the affordances and benefits of the digital format can be exploited more fully. It highlights the value of combining participatory design with visualisation to embed explicit, experiential and even tacit clinical knowledge into the EMR interface.
\end{abstract}

Electronic medical record. Visualisation. Information architecture. Usability. Provotype. Psychology.

\section{INTRODUCTION}

It is not sufficient simply to convert paper records into electronic records because their potential affordances require harnessing in different ways (Gibson 1991). In a health informatics world vulnerable to technological determinism, paper to digital is a complex shift that warrants close, collaborative attention in order to protect the heritage of medical practice.

The role of the modern the Electronic Medical Record (EMR) goes far beyond that of the old paper record, no longer limited to the clinical consultation but expanded into administration, commissioning, service planning, disease surveillance and research (Imison 2016).
However, EMR's key clinical role remains the storage and retrieval of patient information when face to face with the patient during the consultation. This information is in the form of clinician 'notes' (the patient's medical history plus clinical findings, reasoning and actions), laboratory test results, imaging and more. Speed and accuracy in retrieving and recording this information during the consultation is even more critical than in the paper era as it supports the principle of 'data collected at the point of clinical care', the quality of which ripples throughout the health system.

Even though EMR promises so much, deep clinical usability is rarely realised (Millard 2016). Since its adoption in the early 2000s there has been a constant stream of criticism regarding the inability 
of EMR interfaces to achieve anywhere near the level of clinical usability afforded by paper records (Wachter 2016; Elwyn 2014; Tait 1977). Poor usability in turn is widely regarded as having a negative impact on clinical care, time efficiency and patient safety (Viltanen 2011; Shneiderman 2011). Indeed, EMR have been cited as the number cause of physician burnout in the USA (Collier 2017).

At the root cause level, poor usability interrupts the flow and quality of the clinical consultation. Figure 1 shows a schematic of a typical conversation between two people (Pangaro 2008). A clinical consultation has an additional 'loop' - information held within the EMR needs to be accessed at a speed and resolution to match the flow of conversation. This is very rarely achieved, leading to frustration (Csikszentmihalyi 1993), poor clinician-patient engagement and incomplete information assimilation.

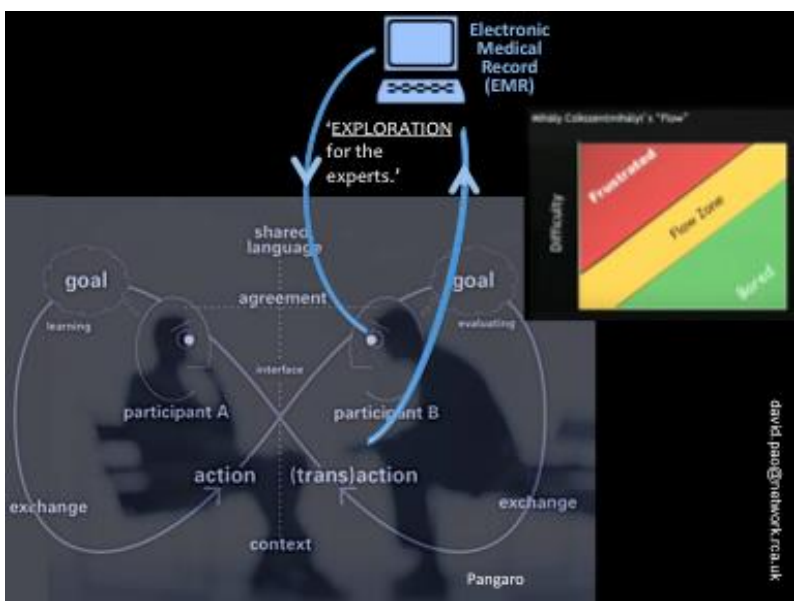

Figure 1: EMR usability affects the flow of the clinical consultation

This disruption in flow comes about despite attempts - most notably by Human Computer Interaction $(\mathrm{HCl})$ - to achieve a satisfactory level of clinical usability. The majority of $\mathrm{HCl}$ research seems to be focused mainly on the analysis of task and process (Zahabi 2015; Zhang 2013). This means most EMR interface research consists of observing clinicians using existing EMR interfaces (Zheng 2009), resulting in interfaces that become highly complex in an attempt to embed clinical knowledge and relevance into an interface that has no clinical 'heritage'.

The research contributions from visual design and visual analytics offer a deeper approach to understanding the needs of the clinical user (West 2015). One of the most useful applications of visualisation in EMR interfaces has been that of the timeline (Aigner 2012; Plaisant 1996). Timelines have the potential to transform our understanding of time-dependent healthcare data. However visual anaytics projects less commonly tackle the entire patient case histories, tending to focus on specific components of it (see discussion, section 6).

This research argues that the clinical user needs an interface that facilitates reasoning as well as task, acknowledging that the clinical consultation represents an exploration of an unknown information landscape as well as the documentation of pre-determined tasks. Achieving this shift means re-imagining the way we approach not only EMR interface design but also the way we look at the clinical consultation itself. This is timely as we move from paper to digital, allowing us to align EMR interface design with the characteristics of the 'machine aesthetic', described by Broeckmann as 'being associated with process rather than object, with dynamics rather than finality, with instability rather than permanence, with communication rather than representation...' (Broeckmann 1997).

All this comes at a time of rapidly increasing access to, and ownership of, data by patients. In a few years' time interfaces will no longer serve the clinician alone - the same data will be accessible by both parties, effectively morphing into some kind of shared platform (Healthcare Intelligence Network 2017). This is when the mantra of 'explanation for the uninitiated, exploration for the experts' will come to the fore (Strausfeld 2014).

For the moment this research focuses on the experts and their need for data exploration, asking how visualisation can be used to present complex medical data rich in clinical context and functionality.

\section{METHODOLOGY}

The lead researcher is a consultant in sexual health with many years' experience using EMR, also undertaking a $\mathrm{PhD}$ in design at the Royal College of Art. Ethical consent was obtained from the Royal College of Art ethics board.

\subsection{EMR usability survey}

A mixed methods paper survey was distributed between January and June 2017 to four sexual health clinic sites using two different types of EMR. Clinicians worked across all clinical roles - doctors, nurses, healthcare assistants and health advisors. Surveys were returned by pre-paid envelope or to a drop box in the clinic.

The survey explored their experience of their current commercial sexual health EMR. It consisted of fourteen 7-point Likert-type statements (11 positive and 3 negative) requiring a graded disagree / agree response, and six 7-point Likerttype statements requiring a graded negatively / 
positively response. These asked about the EMR interface they use in terms of usability as well as impact on self-perceived clinical competence, relationship with patients, autonomy and performance. These statements are detailed in Figure 2.

Respondents were also offered six free text boxes, which invited them to write about their experience using their EMR. These specifically invited exand on how their EMR affected their sense of clinical competence, relationship with patients and autonomy, as well as what they found frustrating, what they liked and how they might design it better.

\subsection{Provotype design}

A provotype is a provocative prototype (Boer \& Donovan 2012), introduced in the design development process to cause a reaction - to provoke and engage people to imagine possible futures. This is different from a prototype, which tends to be closer to the actual representation of a design idea.

Responses to the survey were analysed and categorised, and used to inform the design of the provotype interface (single patient view) consisting of 2 windows (one window is shown in Figure 3a). These were developed using HTML5 and CSS and run on a Firefox web browser. This was driven by a cloud-based database containing fictitious patient data, based on real clinical scenarios but not including any real patientidentifiable information.

All clinical data points essential to the field of sexual health and contraception were included in the database structure and coded into schematics that were displayed on the interface either as an overview (Figure 3a) or episode window. Most of these were as a timeline, some as non-timeline salient data, some as both. Datasets included patient demographics, presenting complaint, history of presenting complaint, examination findings, clinic bedside test results, laboratory test results, blood test results, vaccinations, treatment plans, advice given, surveillance codes, medication, allergies, risk factors, past medical history, obstetric history, family history and psychosocial factors.

\subsection{Provotype evaluation}

The provotype was demonstrated at 4 workshops run at each of the four sites, between August and December 2017. Each session lasted around two hours and consisted of a 'test drive', where between one and three clinicians were asked at random to use the interface, loaded with fictitious patient records, as if the patient was in the waiting room waiting to be seen. The interface was displayed on a large screen while other attendees observed and commented. Each session was audio recorded.

At the end of each session, attendees were asked to complete a shortened version of the paper survey (questions 1a to $1 \mathrm{n}$ only) with questions slightly modified to an 'if I were to use this interface' scenario. Statments are detailed in Figure 4. Qualitative comments were also invited, this time recorded in a single free text box and also by audience participation.

\section{RESULTS}

\subsection{EMR usability survey results}

Results from the 14 Likert statements that required a Disagree / Agree response (*indicates negative statement) and the six statements that required a Negatively / Positively response are displayed in Figure 4, where they are compared to the commercial EMR results.

Qualitative free text responses revealed widespread and consistent dissatisfaction with commercial EMR interfaces, from a clinical usability as well as clinical performance perspective. There were some startling comments such as it makes me feel out of control' and 'quite often, I find myself just guessing'. Whilst negative sentiments covered a broad range of usability and performance areas (often several times), positive sentiments were limited to three areas: no longer losing paper notes, remote site shared access and legibility. These are summarised and compared in Table 1.

Analysis of the survey feedback informed the development of an EMR provotype and included the following functionality / usability features:

1. Low data density: increased visuals, reduced text

2. Getting lost: single window, 2 linked 'anchor' screens

3. Poor consultation flow: mirroring clinical reality, intuitive screen layout, hover-over

4. Poor history overview: timeline overview and semantic zoom for time-dependent data

5. Repeated questioning: data salient data boxes for time-independent data

6. Data density: chunking, glyphs, drill-down for results, vaccinations, risk factors, alerts

7. Results overview: development of results matrix 


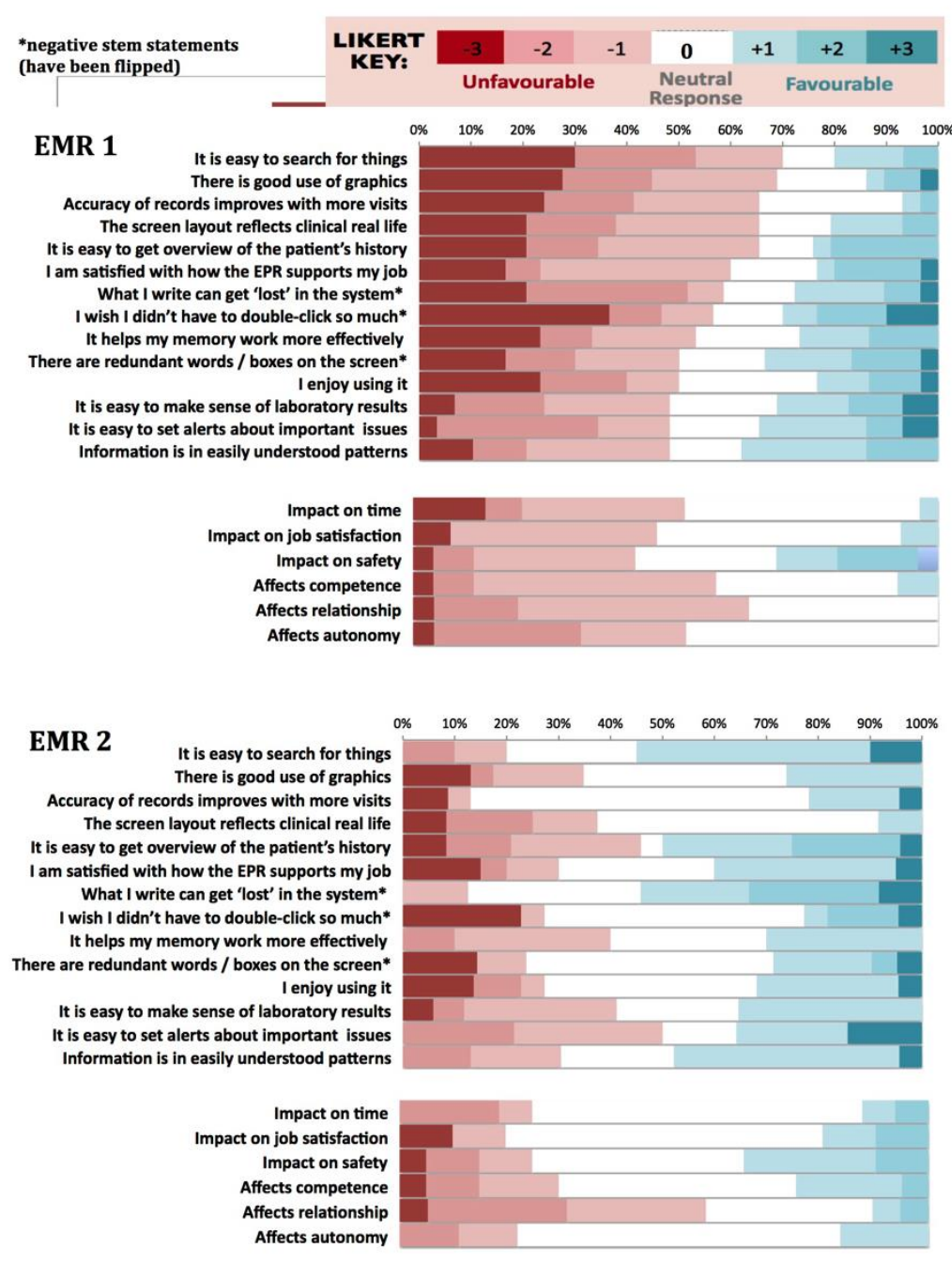

Figure 2: 7-point responses to Likert-type statements ranked in order of degree of deviation from neutral

Table 1: Feedback comments, commercial EMR vs. provotype

\begin{tabular}{|l|l|l|}
\hline Performance & \multicolumn{1}{|c|}{ Commercial EMRs } & \multicolumn{1}{c|}{ Provotype } \\
\hline $\begin{array}{l}\text { Frustration, sense of incompetence, time } \\
\text { inefficiency, poor history overview, poor clinical } \\
\text { relevance, repeated questioning, difficulty with } \\
\text { complex patients, difficulty with patients with } \\
\text { multiple previous visits, impaired flow of } \\
\text { consultation, difficulty maintaining eye contact / } \\
\text { rapport and some even using paper before } \\
\text { transcribing into EMR, not 'losing' records, } \\
\text { benefit of remote site accessibility }\end{array}$ & $\begin{array}{l}\text { Desire to try in a real clinic, 'want this } \\
\text { now', vast improvement, looks brilliant, } \\
\text { highly intuitive, more satisfying, easier, } \\
\text { safer, better engagement, more time } \\
\text { efficient, less repeated questioning likely }\end{array}$ \\
\hline Interface & $\begin{array}{l}\text { Unintuitive information accessibility, low data } \\
\text { density, nonsensical data location, multiple } \\
\text { windows, too much double-clicking, getting lost, } \\
\text { difficulty understanding results, confusing, } \\
\text { difficulty accessing: vaccination history, } \\
\text { treatment history, allergies, test results and } \\
\text { critical alert, legibility }\end{array}$ & $\begin{array}{l}\text { Good history overview, good use of } \\
\text { colour, efficiency of single window, easy } \\
\text { pattern recognition despite complexity, } \\
\text { daunting at first but quick to learn, love } \\
\text { use of glyphs, user-friendly, good } \\
\text { information visualisation, good data } \\
\text { density (not overcrowded), useful salient } \\
\text { information }\end{array}$ \\
\hline Better & $\begin{array}{l}\text { Usefulness of Amazon website,, reference to } \\
\text { other EMR systems, keyboard shortcuts, } \\
\text { calendar alerts, dictation, summary page, } \\
\text { diagrams }\end{array}$ & $\begin{array}{l}\text { Ideas for better contraception glyph, } \\
\text { calendar alerts, signposting map, partner } \\
\text { notification module, value of involving } \\
\text { users }\end{array}$ \\
\hline
\end{tabular}




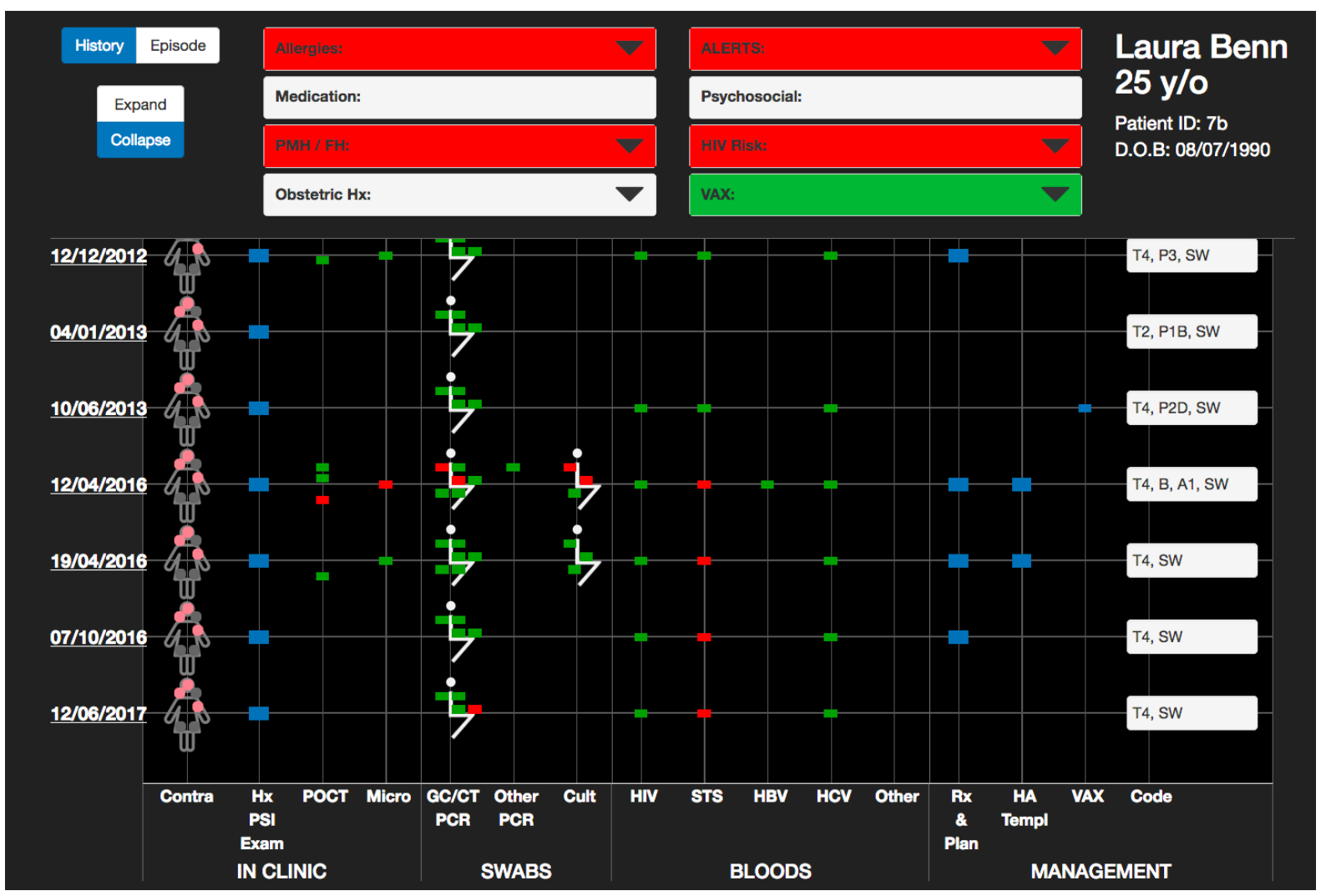

Figure 3a: Overview window

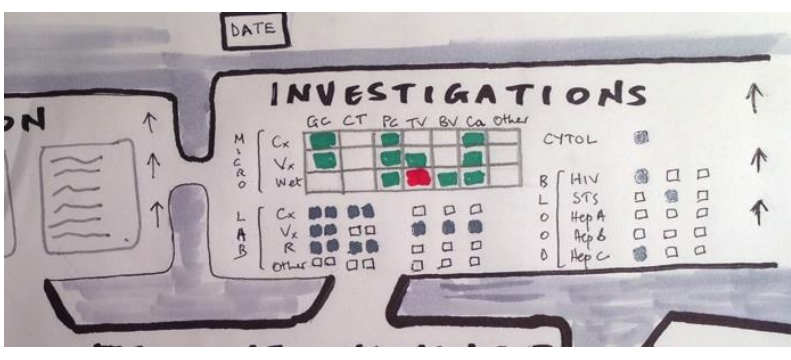

Figure 3b: Sketch of results matrix

\subsection{Provotype evaluation findings}

Four workshops were conducted at four clinic sites. There were between 12 and 22 users at each workshop from the spectrum of job roles in sexual health - doctors, nurses, health advisors and healthcare assistants. Each session lasted around two hours and consisted of a 'test drive', where between one and three clinicians were asked at random to use the interface, loaded with a fictitious patients, as if the patient was waiting to be seen in the waiting room.

The interface was displayed on a large screen while other users watched and commented. Scenarios included typical patient scenarios with multiple previous attendances, with conditions such as HIV, syphilis, gonorrhoea and chlamydia and typical risk factors that affect clinical care.
Responses to the shortened, modified 7-point Likert-type statements are shown in panel 3 of Figure 4. These results show the provotype offers total elimination of negative feedback from ten of the fourteen usability statements and improvement on the remaining four. Limitations and caveats to this observation are described in section 4 .

Free text responses to the provotype interface were limited to a single comments box. In contrast to commercial EMR experience, these sentiments were overwhelmingly positive, for example 'really helpful and efficient' and 'this looks brilliant, I would love to use it' (see Table 1 for comparison).

\section{LIMITATIONS}

There were several potential biases in this study. First, the lead researcher and designer of the initial provotype is a practising doctor. This means that whilst the initial provotype layout had the advantage of being highly clinically relevant it was also idiosyncratic. On balance, it was felt advantageous to start with one specific layout rather than 'design by committee'.

Secondly, the way in which commercial EMRs were evaluated differed from that of the provotype. With the former, the survey evaluated long-term daily use over a wide range of real clinical 


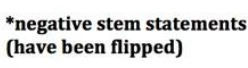

\section{EMR 1}

It is easy to search for things
There is good use of graphics Accuracy of records improves with more visits The screen layout reflects clinical real life It is easy to get overview of the patient's history I am satisfied with how the EPR supports my job What I write can get 'lost' in the system* I wish I didn't have to double-click so much* it helps my memory work more effectively There are redundant words / boxes on the screen* It is easy to make sense of laboratory results It is easy to set alerts about important issues Information is in easily understood pattern

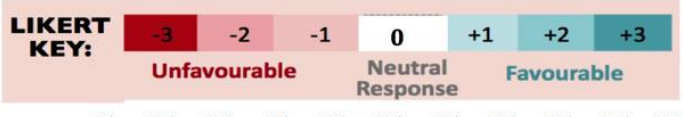

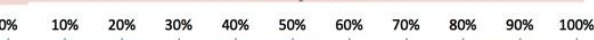

EMR 2
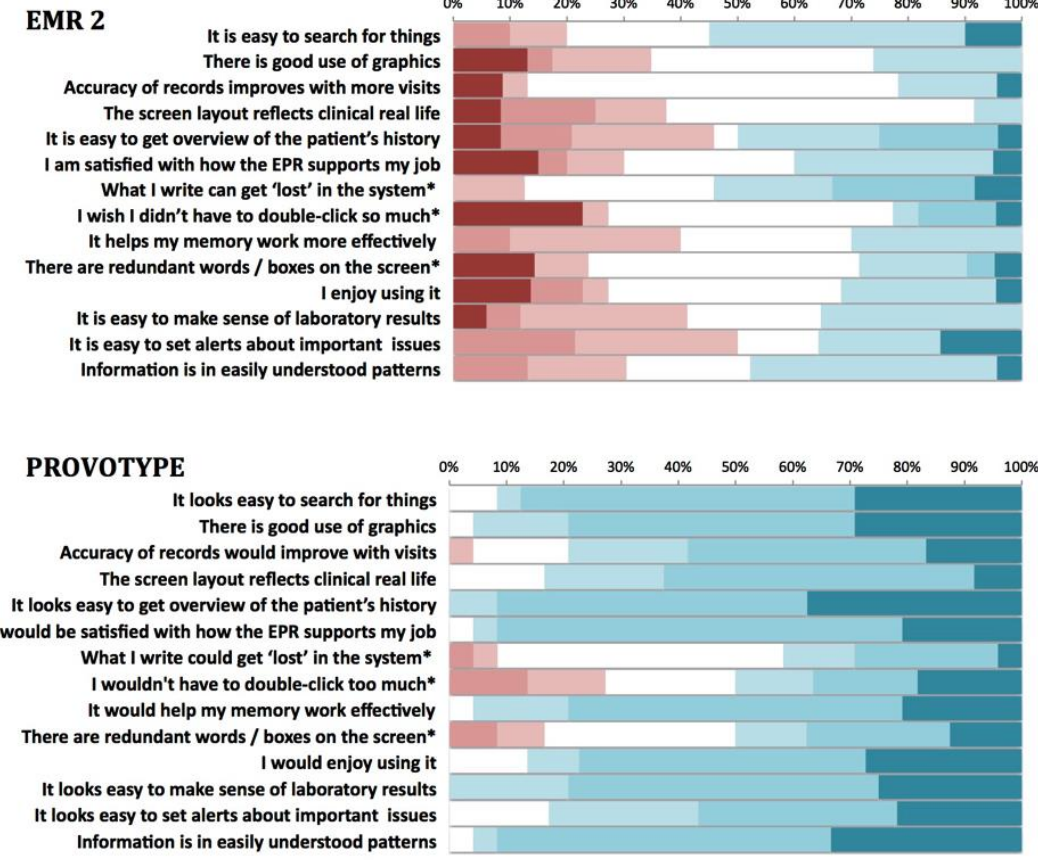

Figure 4: 7-point responses to Likert-type statements ranked in order of degree of deviation from neutral

scenarios. For the provotype, a similar but shorter survey was used, based only on a 90-minute observation of clinical scenario 'test-drives'.

Thirdly, the users and lead researcher are known to each other. Whilst every effort was made to ask the users to evaluate the provotype on merit alone and not to consider the feelings of the researcher / designer, objectivity in responses may have been affected.

Whilst these factors introduce bias, this was felt to be acceptable because the provotype's primary aim was to provoke feedback and discussion rather than to be evaluated. Conversely it was felt that the lead researcher's medical experience was invaluable in the workshop discussions, allowing rapid and coherent discussion around specific topics that required in-depth clinical knowledge. A more objective way of carrying out the research would have been to use a second clinician / designer, but no such individual could be found.

\section{CONCLUSION}

This research highlights the deep dissatisfaction felt by the users of two commercial sexual health EMR interfaces in terms of usability and the subsequent impact on self-perceived clinical competence, relationship with patients, autonomy and performance. This is seen in both the quantitative Likert-type evaluation as well as the feedback comments.

The provotype design was informed by this feedback, drawing on visual design to embed these clinical needs and frustrations into the interface. Evaluation showed the value of this participatory design process, almost eliminating negative usability and performance scores and drawing 
overwhelmingly positive feedback. Statistical analyses will follow in a future paper.

We suggest that this initial approach through provotype design offers a worthwhile starting point for future interface design. The next step would be to develop the provotype in more depth, perhaps re-thinking the initial layout in consultation with users. In sexual health, there are specific areas that would benefit from further visualisation - in particular data concerning contraception, partner notification and risk assessment for sexually transmitted infections. As a starting point, unpublished feedback from audio recordings of each workshop could be used. If applied to other medical specialilties there would be different areas of focus, but the same design principles would apply and the same benefits should emerge.

The subsequent step would be to take a commercially available database 'backend' and develop a bespoke overlaid visual interface that can be tested and evaluated in the field, using standard techniques such as screen capture, eye tracking and 'lab-in-a-box'-type observational tools (Weibel 2016). This would provide evidence for the effectiveness of this approach and fill in the large knowledge gap that exists around how to improve the clinical usability of EMR interfaces.

\section{DISCUSSION}

The lead researcher for this project is both a practising doctor and design researcher. He has personal, experiential and professional knowledge of using EMR in clinical settings and understands the principles and practice of medicine at a professional level. Whilst this introduces bias (see section 4) it also represents a valuable perspective that bridges the gulf between clinical and design silos.

The EMR can be thought of as the cornerstone tool of clinical medicine. It is difficult to think of a professional discipline other than medicine where interface design offers such poor usability and provokes so much dissatisfaction. In 2016 the Nuffield Trust reported that "health care is at least a decade behind other industries in the use of information technology' (Imison 2016).

Many commercial EMR interfaces begin their life as some kind of non-clinical (typically management or pathology) software platform, or at least display those technological characteristics. This research explores a novel approach that puts the principles and practice of clinical practice over and above any interface convention or paradigm.
There is no doubt that medicine represents a highly complex data landscape and a profession that is highly sensitive about patient safety. This is probably why EMR evolution is so conservative.

Unburdened by these constraints, we challenge the fundamental dogma that the clinical consultation should primarily be seen as task. We assert that while task is important, the primary information landscape of the consultation is one of data exploration. This research aims to make sense of data complexity and at the same time mitigate risk by designing for deep clinical relevance from the very earliest stages of interface design.

To achieve this, data need to be represented in a way that mirrors clinical reasoning - and this needs visualisation. It is for this reason that some of the best research comes from the visual analytics community (West 2015; Rind 2013). However it is rare to find a visual analytics project that tackles the entirety of the patient's case - most focus on specific areas. Excellent examples of these include glucose visualisation (Powsner 1994), medication reconciliation (Plaisant 2015) and intensive care visualisation (Horn 1998).

At its core, this research aligns with the widely held view that EMR usability impacts the quality of clinical care (Middleton 2013) but argues for a broader, more clinically-oriented definition of usability. It asks for a re-imagining of the way we approach data visualisation, not just in the way we view the clinical consultation (reasoning over task) but also by acknowledging that the relationships between data are as important as the data themselves.

Equally importantly this research suggests a change in the way we collaborate when designing interfaces, highlighting the value of a participatory design approach that includes design, medicine and the visual arts. We feel this approach offers the most potential to embed explicit, experiential and even tacit clinical knowledge into an interface.

If this approach leads to better usability (as this research demonstrates) and higher self-perceived clinician competence, relationship with patients, autonomy and performance (as this research suggests), this in turn will lead to higher quality, safer clinical care.

It will also lead to healthier clinicians, which is now considered one of the 'quadruple aims' of modern healthcare alongside population health, patient experience and cost effectiveness (Bodenheimer 2014). This can only be a good thing - after all, we should be able to judge the health of a system by the health of its workers. 


\section{REFERENCES}

Aigner, W. (2012) Challenges of Time-oriented Data in Visual Analytics for Healthcare. IEEE VisWeek Workshop on Visual Analytics in Healthcare. Utah, USA, 24-29 October.

Bodenheimer, T. et al. (2014) From Triple to Quadruple Aim: Care of the patient requires care of the provider, Annals of Family Medicine, 12(6): 573-6.

Boer, L. and Donovan, J. (2012) Provotypes for Participatory Innovation. Proceedings of the Designing Interactive Systems Conference. Newcastle, UK, 11-15 June.

Broeckmann, A. (1997) Remove the Controls Machine Aesthetics. V2_Lab for the unstable media conference. Rotterdam, the Netherlands, 27 June13 July.

Collier, R., (2017) Electronic health records contributing to physician burnout. Canadian Medical Association Journal, 189(45): E1405E1406.

Csikszentmihalyi, M., et al. (1993) The measurement of flow in everyday life: toward a theory of emergent motivation. USA: University of Nebraska Press.

Elwyn, G (2014) 'Patientgate' - Digital Recordings Change Everything, British Medical Journal, 348: 2078.

Gibson, J. (1981) The Ecological Approach to Visual Perception. Boston, USA: Houghton Mifflin.

Horn, W. et al. (1998). Metaphor Graphics to Visualize ICU Data Over Time. Intelligent Data Analysis in Medicine and Pharmacology. UK: Springer.

Imison, C., et al. (2016) Delivering the Benefits of Digital Health Care. UK: The Nuffield Trust

Middleton, B., et al. (2013) Enhancing Patient Safety and Quality of Care by Improving the Usability of Electronic Health Record Systems: Recommendations from AMIA. Journal of the American Medical Informatics Association, 20(e1): 2-8.

Miliard, M. (2016). Frustrations Linger Around Electronic Health Records and User-centred Design. Healthcare IT News.

http://www.healthcareitnews.com/news/frustrationslinger-around-electronic-health-records-and-usercentered-design (retrieved March 19 2018).

Pangaro, P. (2008) Instruction for Design and Designs for Conversation. In: Handbook of Conversation Design for Instructional Applications. New York, USA: ICI Global Publishers.
Plaisant, C., et al. (2015) Novel User Interface Design for Medication Reconciliation: An evaluation of Twinlist. Journal of the American Medical Informatics Association, 22(2): 340-9.

Plaisant, C. et al. (1996) LifeLines: Visualizing personal histories. Proceedings of the SIGCHI Conference on Human Factors in Computing Systems, 221-7. Montreal, Canada 22-27 April.

Powsner, S. M., et al. (1994) Graphical summary of patient status. The Lancet, 344(8919): 386-9.

Rind, A. (2013) Interactive Information Visualization to Explore and Query Electronic Health Records, Foundations and Trends ${ }^{\circledR}$ in Human-Computer Interaction, 5(3): 207-98.

Shneiderman, B. (2011) Tragic Errors: Usability and Electronic Health Records. Interactions, 18(6): 60-3.

Strausfeld, L., (2014) Visualising Data. Lecture at Stamps School of Art \& Design, Michigan University, MI, USA.

Tait, I. (1977) The History and Function of Clinical Records (MD Thesis). UK: University College London.

Viitanen, J., et al. (2011) National Questionnaire study on Clinical ICT Systems Proofs: Physicians suffer from poor usability. International journal of medical informatics, 80(10): 708-25.

Wachter, R. (2016) Making IT Work: Harnessing the power of health information technology to improve care in England. London, UK: Department of Health.

Weibel, N. (2015) LAB-IN-A-BOX: semi-automatic tracking of activity in the medical office. Personal and Ubiquitous Computing, 19: 317-334.

West, V. (2015) Innovative Information Visualization of Electronic Health Record Data: A systematic review, Journal of the American Medical Informatics Association, 22(2): 330-9.

Zahabi, M., et al. (2015) Usability and Safety in Electronic Medical Records Interface Design: A Review of Recent Literature and Guideline Formulation. Human factors, 57(5): 805-34.

Zhang, Z., et al. (2013) The Five Ws for Information Visualization with Application to Healthcare Informatics. IEEE transactions on visualization and computer graphics, 19(11): 1895-910.

Zheng, K., et al. (2009). An Interface-driven Analysis of User Interactions with an Electronic Health Records System. Journal of the American Medical Informatics Association, 16(2): 228-37. 